\title{
LA PROPORCIÓN MEDIEVAL EN EL DISEÑO Y CONSTRUCCIÓN DE LA CATEDRAL DE TORTOSA
}

\author{
JOSEP LLUIS I GINOVART ${ }^{1}$ \\ Universitat Rovira i Virgili
}

\begin{abstract}
La construcción gótica está sujeta al saber del magister operis, condicionado en el contexto de las catedrales, por el Capítulo. El ábside de la catedral de Tortosa es un banco de pruebas, y en este entorno aparece la traza de Guarc (c.1345-1380). El análisis del pergamino descubre los métodos de trazado del ábside con la figura del heptágono, y del cimborrio con el octógono. El análisis de los códices ACTo 80 y ACTo 236, con obras de Calcidio, Capella y Macrobio, permite conocer la teoría de la que emanaba la proporcionalidad numérica que los canónigos conocían.
\end{abstract}

Palabras clave: Catedral; Geometría medieval; Diseño medieval; Catedral Tortosa.

\section{MEDIEVAL PROPORTION IN THE DESIGN AND CONSTRUCTION OF TORTOSA}

\section{CATHEDRAL}

Gothic construction is subject to magister operis knowledge, conditioned in the context of the cathedrals by the Chapter. The apse of Tortosa Cathedral is a testing ground, and in this context the Guarc trace (c.1345-1380) appears. Analysis of this parchment reveals the design method used for the apse, with the figure heptagon, and the dome, with an octagon. Review of the codices ACTo 80 and ACTo 236 with the works of Calcidius, Capella and Macrobius, reveals the theory behind the numerical proportionality known by the Canons.

Key words: Cathedral; Medieval geometry; Medieval design; Tortosa Cathedral.

\section{Geometría y diseño de las trazas góticas}

En el Archivo Capitular de la catedral de Tortosa (ACTo), existe un pergamino $(917 \times 682 \mathrm{~mm})$ que representa la planta de una catedral, con la anotación "En Antony Guarc". En el verso "Fábrica -N. ${ }^{4} 49-$-, "Mostra d'En Antony Guarç"(fig. 1). El documento permite completar los conocimientos geométricos de los constructores medievales, a la vez que plantear el proyecto gótico en los términos de diseño medieval propuestos por Roland

\footnotetext{
1 josep.1luisg@urv.cat
} 
Recht (1941- $)^{2}$. La metodología utilizada es complementaria a los estudios específicos del ms. 1092 Stiftsbibliothek, del plano de Sant Gall (c.820), de Walter Horn y Walter Born ${ }^{3}$. Se realiza a través del análisis de los elementos auxiliares de trazado. La renovada búsqueda del canon gótico en la geometría platónica, planteados por Nigel Hiscock (2000) y Robert Bork $(2011)^{4}$, permiten inducir una teoría proporcional en la catedral que Guarc había de construir La introducción desde el análisis informático en la geometría gótica de Dag Nilsen (2001) y Han Vandevyvere (2001), junto la revisión de las geometrías prácticas desde de la historia de matemática de Jenes Høyrup (2009), son los antecedentes metodológicos en la exploración del pergamino ${ }^{5}$.

El interés del dibujo gótico, y su adecuada catalogación, tiene como antecedente el proyecto de Francois Bucher en el Architector. The Lodge books and sketchbooks of medieval architects (1979), pretendiendo recorrer así, desde Villard de Honnecourt, hasta Rodrigro Gil de Hontañon. A estos hay añadir los de la exposición de Les batîsseurs de cathédrales gothiques en Strasbourg (1989), con las principales trazas europeas editadas por Roland Recht (1941-). En España el intento de recopilación de las principales referencias góticas y tardogóticas ha sido realizado por Begoña Alonso Ruiz (1966-) y Alfonso Jiménez Martín (1946-)6, convirtiendo la traza de Guarc en la más antigua conservada sobre pergamino en España.

\section{La Mostra d'En Antony Guarc}

El pergamino de Guarc fue transcrito por José Matamoros (1932) y recogido por Pierre Lavedan (1935)7, modificado en 1984 por Josep Lluis (1958-), publicado también en 1991 por Victoria Almuni (1959-). ${ }^{8}$ El primer estudio sobre la traza, atendiendo a la estructura modular del gótico fue, La traça de la catedral de Tortosa. Els models d'Antoni Guarc i Bernat Dalguaire (1997), de Almuni y Lluis. La traza será divulgada en Sancta Maria Dertosae. Guía de la catedral (2000) ${ }^{9}$. La figura del maestro Guarc será contextualizada por Almuni, en La catedral de Tortosa als segles del gòtic (2007) ${ }^{10}$. Otras aproximaciones desde la geometría medieval han sido publicadas en los catálogos de las exposiciones; Fidei Speculum (2000), La Llum de les Imatges (2005), y en la Traça de la iglesia de Sevilla ${ }^{11}$.

Guarc representa la planta de un proyecto de cabecera y parte del cuerpo de la nave de una catedral. El sistema de representación del conjunto es de proyección ortogonal, ichonographia, excepto en el detalle de la puerta de la capilla anexa, representada como proyección vertical. Matamoros sitúa el plano de Guarc, en los preparativos de la construcción gótica, antes de la intervención de Bernat Dalguaire y Benet Basques de Montblanc (1345). Almuni lo hace en la reanudación de la fábrica hacia (1375), con la aparición onomástica de Guarc, en el los libros de fábrica (1379-1382). Así la cronología de la traza ha de situarse entre (1345-1380) ${ }^{12}$.

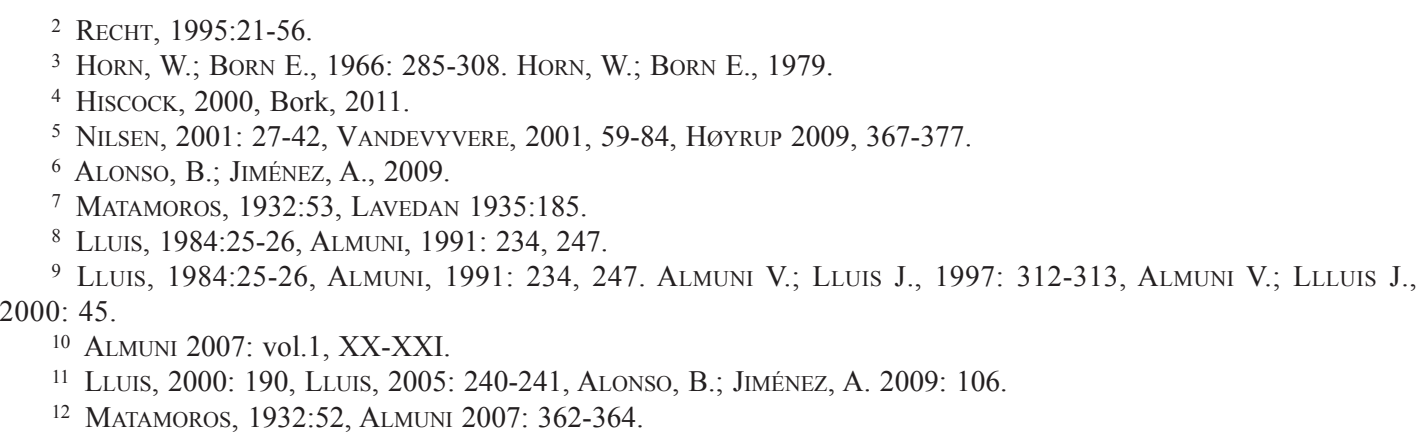



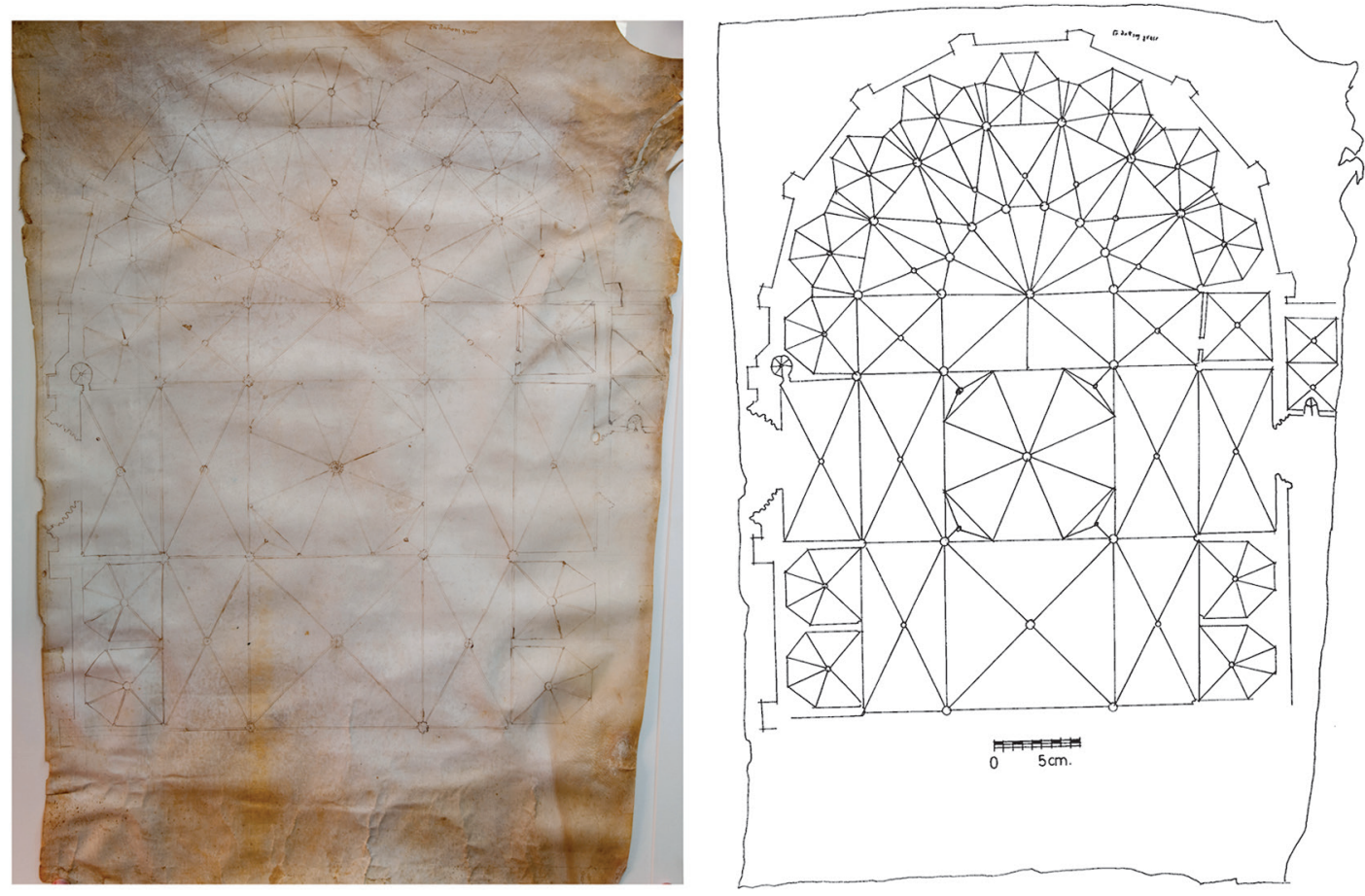

Fig. 1. Pergamino Antoni Guarc (c.1345-1380). ACTo Fábrica 49.

La representación gráfica de plantas góticas, como la de Guarc, son escasas, debido a la abstracción geométrica que ello requiere. Las existentes están concebidas como anotaciones tipológicas, como las del Villard de Honnecourt (c.1175-1240) (BNP ms.fr.190093), en Notre-Dame de Cambrai (fol.14v.), la catedral de Saint-Etienne de Meaux (fol.15r), y la abadía de Vaucelles $(\text { fol.17r })^{13}$. Otras son realizadas como traslados de modelos; las atribuidas a Michel de Fribourg, de las catedrales de París y de San Croix de Orleans (1388) (Musée de l'Oeuvre Notre-Dame de Strasbourg, Inv.29, Inv. 21) ${ }^{14}$. Otras trazas se plantean como un proyecto global de edificio; Steyr (Vienna Akademie ABK 17052), Zagreb (ABK 16926), Augsburg (ABK 16846), Kuttemberg $\left(A B K 1_{16841}\right)^{15}$, y la de Nürnberg (German-isches National-Museum) ${ }^{16}$. En Italia existen, el proyecto de la Catedral de Milán atribuido a Henri Parler (c.1392) (Musée de l'Oeuvre NotreDame de Estrasburgo, Inv.n. $\left.{ }^{\circ} 29\right)$, y el de Antonio de Vicenzo (1390-1392) (Museo de San Petronio de Bolognia, cart.389, n. ${ }^{\circ} 1$ ), completados con los diseños (S2-S3) del Duomo de Siena (Museo dell’Opera della Metropolitana) ${ }^{17}$. En la Península Ibérica, las trazas tardogóticas son posteriores a Guarc, como la de la catedral de Sevilla, del Convento de Bidaurreta (1433?,

\footnotetext{
13 Lassus, 1858: 112-124,130-131. Bucher 1979: 96-107. BechmanN, 1993: 108-112.

14 BuCHER, 1968: 59, fig.15, VANDEKERCHOVE, 1989: 317-318.

15 BUCHER, 1968: 59.

16 BUCHER 1972: 38.

17 AsCANI, 1989: 268-270, AsCANI, 1997: 89-94.
}

Arch. esp. arte, LXXXVII, 348, OCTUBRE-DICIEMBRE 2014, 321-334 ISSN: 0004-0428, eISSN: 1988-8511, doi: 10.3989/aearte.2014.20 
1490 ? $)^{18}$, el pergamino de Bartolomé de Pelayos para la catedral de Coria $(1502)^{19}$, la Planta de la Iglesia de San Bartomé de Javea $(1513)^{20}$, la de Juan Gil de Hontañón en la de Segovia $(1524)^{21}$, o las de Juan de Alava en el Convento de San Esteban de Salamanca (1524) junto con el de la catedral de Salamanca (c.1537) ${ }^{22}$.

\section{Geometria fabrorum en la traza de Guarc}

El estudio del documento permite el análisis de los medios auxiliares de trazado, líneas y puntas de compás, sobre la que se traza la planta definitiva, y así de las cuestiones definidas como geometria fabrorum ${ }^{23}$. Sobre el soporte existen unos orificios que penetran el pergamino, unos en el perímetro fijándolo (Pa1), y otros de puntas de compás. En ellos cabe distinguir los del transporte de medidas ( $\mathrm{Pa} 2)$, y los que trazan la circunferencia, que rasgan la superficie (Pa3). El análisis de los puntos da la secuencia del trazado, y determinan el número de operaciones que parte de él. Hay puntos que tienen tan sólo una solicitación, hasta otros PO.1, con cinco utilidades. En este punto se concentra la mayoría del trazado auxiliar, reconociendolo como punto de origen. Las líneas están trazadas con punzón, en los tramos rectos (La4), o con compás de punta seca, en las secuencias circulares (La5). En cuanto a la técnica gráfica de líneas: unas trazadas con elementos punzantes que alterarán la superficie; y otras semejantes al trazo de grafito. Las primeras son las que encajan el dibujo y trazan las proporciones, mientras que las segundas son líneas auxiliares, y fijadas con trazos definitivos de tinta (La6) (fig. 2). El encaje del pergamino se realizó mediante un modulo inicial. Así se divide el ancho de la catedral en 6 partes $(91 \mathrm{~mm})$, unidad patrón. El rectángulo junto a la clave del presbiterio tiene una relación de $(91 / 82 \mathrm{~mm})$, de manera que la proporción entre el ancho de las capillas laterales y el muro de separación es de 8/1, teniendo el módulo de la nave colateral 9 y la central 18 unidades.

La secuencia de trazado permite determinar el método geométrico del trazado del octógono del cimborrio, y la del polígono de 14 lados derivado del heptágono del ábside. El cimborrio se diseña a partir del cuadrado de base, abatiendo su semidiagonal (fig. 3). Es el mismo trazado que el publicado en la Geometrie Deutsch (1488) (fol 3r.) de M. Roritzer (1440-1493) ${ }^{24}$. Este método difiere de las fuentes gromáticas del Fragmentum de hexagono et octogono atribuida a M. T. Varro (116-27aC) $)^{25}$ y de los de tradición árabe de Abū al-Wafā Al-Būzjānī (940-998) Kitāb fì mā yahtāju al-ṣāni 'min al-a 'māl al-handasiyya (c.993-1008) (Chap.II.8)26. También es diferente del la figura mediatrix de De triangulis (c.1250) de Jordanus Nemonarius (1225-1260) $\left(\right.$ P.IV.15) ${ }^{27}$.

Para trazar el doble heptágono del ábside, Guarc realiza una operación previa. Sobre el diámetro del presbiterio de módulo 18, abate la medida que va a tener la capilla radial de 8 módulos. En definitiva, Guarc utiliza la relación numérica 18/8 entre la nave central y la capilla lateral, o

18 AlOnso, B.; JimÉNEZ, A., 2009b: 63-74.

19 SÁNCHeZ, 1982: 63-76.

20 ARroyas M., ZaragOZÀ A., 2003:175-176.

21 CASASECA, 1978: 29-51.

22 Alonso, B.; JimÉnEZ, A., 2009: 116-117.

${ }^{23}$ Ruiz de la Rosa, J. A., 1987a: 52-59. Ruiz de la Rosa, J. A., 1987b: 195-208.

24 Roriczer, 1999: 56-60, Heideloff, 1844: 97, HoffStadt, 1847: 20, Shelby 1977: 119-120, Recht 1980:25.

25 BuBNOV, 1899: 552.

26 WoEPCKe, 1855: 330, RAYNAUd, 2012: 34-83.

27 Octogonus circulo inscriptus inter quadratum eidem inscriptum et quadratum circumscriptum proportionali. Curtze, 1887, Beaujouan, 1975: 453-454. 


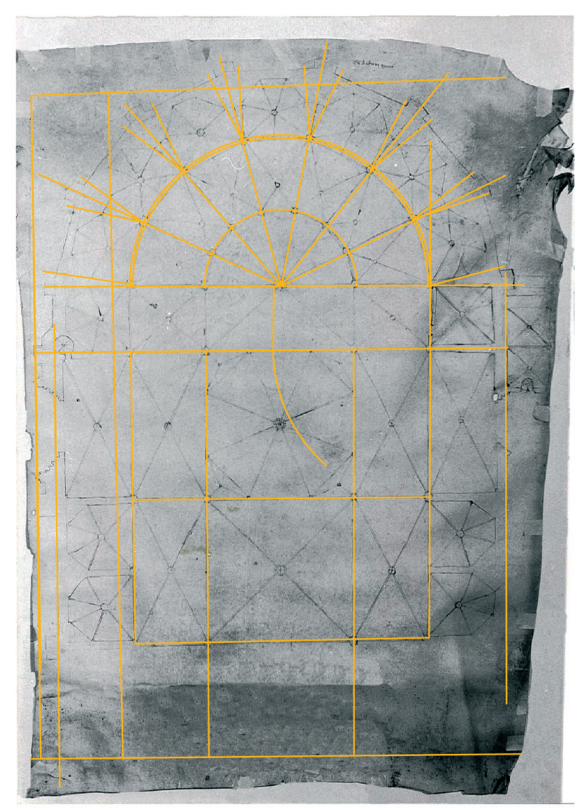

1

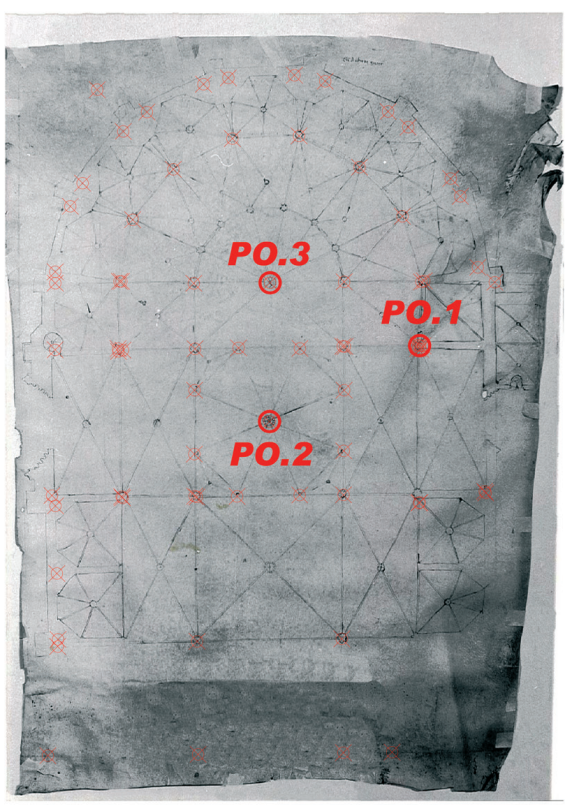

2

Fig. 2. Trazos auxiliares del pergamino de Guarc; (1) líneas, (2) puntos.
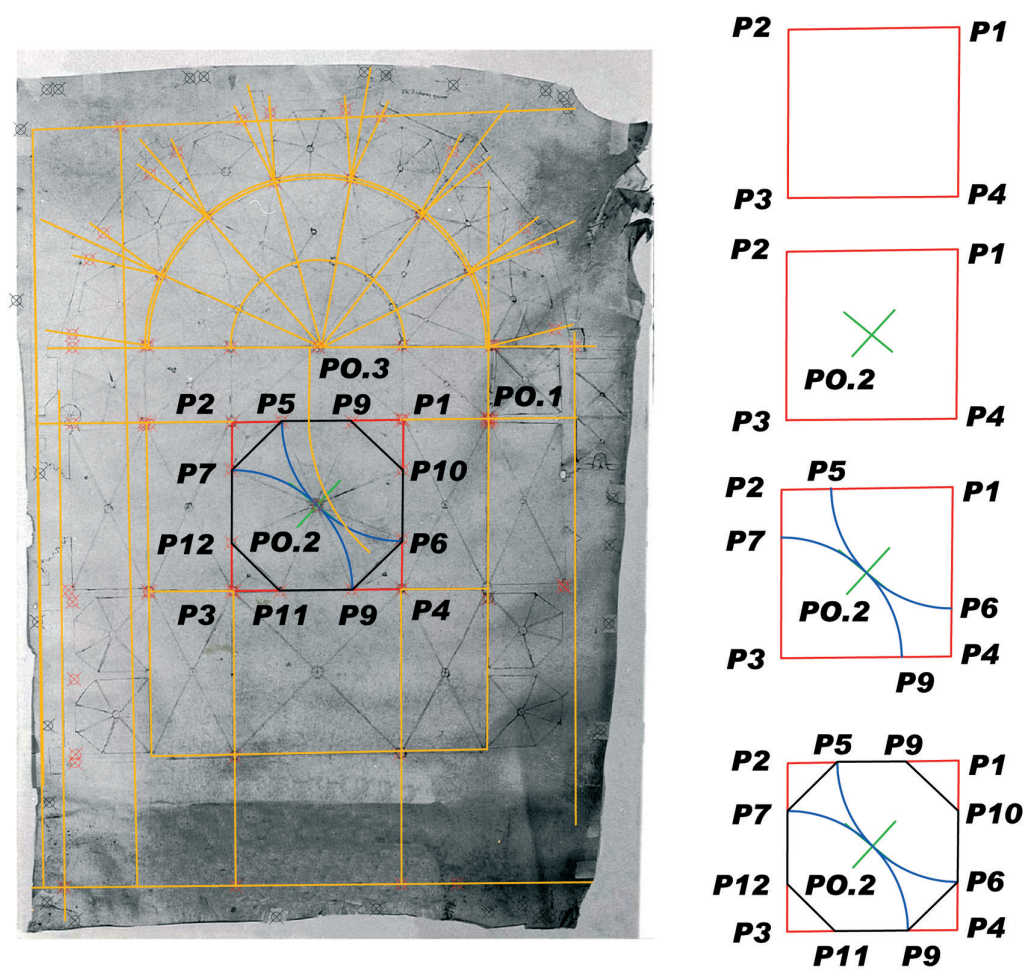

Fig. 3. Trazado del octógono en el pergamino de Guarc.

Arch. esp. arte, LXXXVII, 348, OCTUBRE-DICIEMBRE 2014, 321-334 ISSN: 0004-0428, eISSN: 1988-8511, doi: 10.3989/aearte.2014.20 
lo que es lo mismo 9/8 entre el ancho deambulatorio y la capilla radial. El método difiere de la Geometrie Deutsch (1488) de Roriczer, en la que la construcción se realiza mediante una operación combinada de compás, a partir de altura del triángulo equilátero, de lado el radio de la circunferencia ${ }^{28}$. En el tardogótico se traza el heptágono a partir de la altura del triangulo equilátero de base el radio. De esta manera lo hace Charles Bouvelles (1478-1567) Libellus de mathematicis supplementis (1509) (Mas. Sup. fol.192) y A. Dürer (1471-1528) en Underweysung der Messung (1525) (L II.12, 62), mediante el corolario del pentágono (L II.15, 62). A partir de aquí estas soluciones tardogóticas tienen la misma base geométrica, y cuyo origen está en el libro de construcciones geométricas necesarias para los artesanos de Abū al-Wafā Al-Būzjānī ${ }^{29}$. Los métodos geométricos góticos y tardogóticos difundidos en los tratados, están lejos del utilizado por Guarc (c.1345-1380). Tan sólo el Manifiesto Geométrico (1684) de Fray Ignacio Muñoz (1612-1685), determina una solución parecida con la relación de 4/9 entre el lado del heptágono y el radio.

\section{Las fuentes directas de los promotores de la catedral de Tortosa}

El estudio pormenorizado de los códices del Archivo Capitular de Tortosa ACTo, más allá de la catalogación de Bayerri (1962), ha permitido identificar algunas fuentes directas de obras que abordan la proporcionalidad numérica.

El Civitatis Dei ACTo 20 de San Agustín (354-420) hace referencia a la teoría numérica. En el libro XI (fol. 156r.-170v), dice que el seis es la perfección; así es el primer número que resulta de sus partes, $6=3+2+1$ (XI.30). El número 10 , es el que tiene como divisores a $(5,2,1)$, mientras que el 12 tiene a $(6,4,3,2,1)$ (XI.30). El siete representa el séptimo día, se reconoce como el reposo del Señor, 7=4+3 (fol 168v-169v) (XI.31) ${ }^{30}$. En el libro XX (fol.333r-359r), el número doce, $3 \times 4=12$. El doce es el triple de cuatro y cuádruplo de tres (fol. 335r-336v) (XX.5.3). El mil es el número perfecto la plenitud del tiempo. Es la figura cuadrada, que es plana, y al darle altura, se hace cúbica (fol. 337v-338v) (XX.7.2) ${ }^{31}$.

Otro texto es el Timaeus de Calcidio (f.350) ACTo 80 (146r-155v.14), del siglo XII, acompañado en el (fol.150r.) por uno de sus comentarios (XXXII) ${ }^{32}$ (fig. 4). En él aparece la secuencia numérica $(1,2,4,8)$ y $(1,3,9,27)$, con las modulaciones armónicas (XLI). Así se generan: el uno; el dos $(2=2 \times 1)$; el tres $(3=2+1 / 2 \times 2)$; el cuatro $(4=2 \times 2)$; el ocho $(8=1+7)$; el nueve $(9=3 \times 3)$ y el $27(27=27 \times 1)$. Otras proporciones son la parte más su medio $(1+1 / 2)$; la parte y su tercio $(1+1 / 3)$, llamada epítrito; y la parte más su octava $(1+1 / 8)$, epogdo. Aparece el duplo, el triple y el cuádruplo, además de la relación (243:256). En el (fol.155v.15-66) un Comentario del Timeo (XLIX), referente a la proporcionalidad de las modulaciones numéricas, la diatesarón y la diapente. Inserto en el ACTo 236 (fol.39), el Comentario (II,1,15) del Timeo de Calcidio, con el esquema de las relaciones armónicas.

Otra referencia es el Comentarii In Somnium Scipionis del ACTo 236 (fol.1-61v) de Macrobio (f.400) de siglo XIII ${ }^{33}$ (fig. 4). Define el esquema de la secuencia $(1,2,4,8)$ y $(1,3,9,27)$, recordando el dios creador del alma, tomando números pares e impares con dobles y triples (fol. 6v 19-

28 Roriczer, 1999: 56-60, HeIDEloff, 1844: 95-99, HofFSTADT, 1847: 17-18, SHELBY, 1977: 118-119, RECHT 1980: 24-25.

29 WoepCKE, 1855, 218-256: 309-359 (CII.6) y (CIII.13)

30 Agustín, 2000: (XI.30) 742-743,

31 Agustín, 2001: (XX.5.3) 644, (XX.7.2) 655.

32 La notación edición WASZINK. 1975.

33 La notación edición de WILLIS, 1970. 

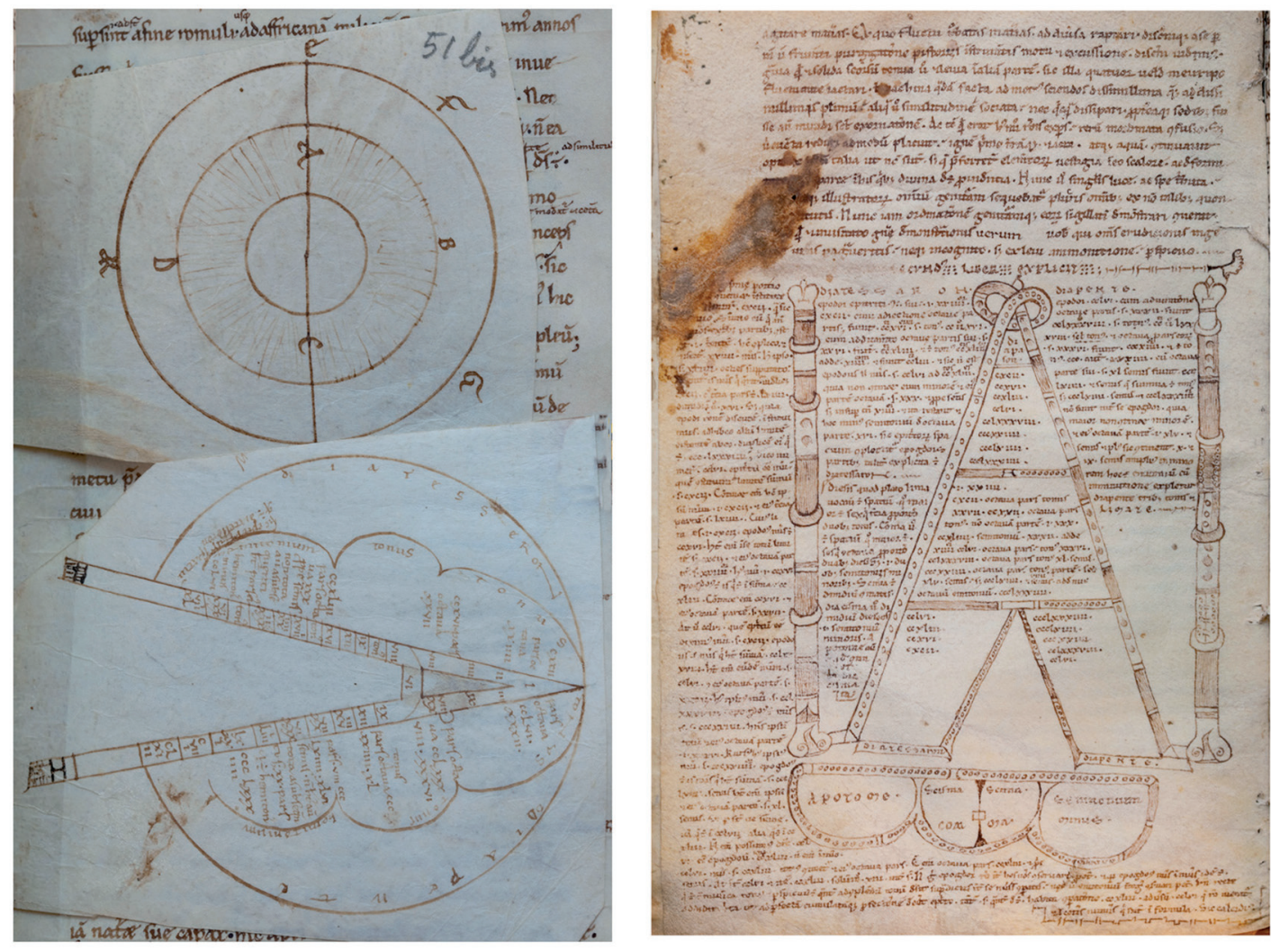

Fig. 4. Fuentes neoplatónicas. Macrobio ACTo 236, Calcidio ACTo 80.

22). En el libro segundo define la armonía de las esferas (II,1,1). También el porque oímos la música de la esferas (II,4,15), comentando la relación (243:256) (II,1,15-22), a la que los pitagóricos llamaron diesis.

Por otra parte, un fragmento de la Geometría del Libro VII del De Nuptiis Philologiae et Mercurii de Marciano Capella (f. 430), ACTo 80 (fol. 160 v.28- fol. 161r.5) del siglo XII, define los géneros de las figuras planas a las que llama ergásticas. También las líneas rhētós, o álogos; la primera línea es racional, y puede ser comparada, en cambio la segunda no puede ser cotejada. Las líneas son isotếs cuando la proporción de dos de ellas, tiene una longitud igual o doble. Se dice homólogos cuando éstas son coincidentes. Análogos, cuando la línea es superada por otra en el doble de longitud, y supere a su vez, a una tercera en la misma proporción. La línea álogos, o irracional, es la que no coincide ni en igualdad ni en la mitad o en la tercera parte, ni en el doble, ni en el triple, ni tampoco en ninguna otra cantidad ${ }^{34}$.

Aparece la Geometria Incerti Auctoris de Gerberto de Aurillac (c. 940-1003). ACTo 80 (fol. 159r.1-160v.27), siglo XII, con 20 proporciones del L.III y L.IV ${ }^{35}$. Aborda cuestiones relacionadas con la medida indirecta de objetos, con fuentes diversas. Las proposiciones P1 y P2 tienen

${ }^{34}$ Edición de WiLlis, 1983. Ergasticis Schematibus [715]... t, quarum prima dicitur Mese Alogos [720].

35 BuBnOv, 1899: 317-330, 336-338. 
fuentes gromáticas; las P3, P4, P5, P6, P7, son utilidades del astrolabio de origen árabe; mientras que las P8 y P9, tienen fundamentos en la óptica. Otras como las P18, 19, 20 i 21 pertenecen a la Óptica Pseudo-Euclides, así como las P10, P11, P13, P15 y P16. La proporcionalidad del triangulo, P12, P14, P17, y P20, con la escuadra isósceles P18, y la pitagórica P19. Las proposiciones tienen una metrología de proporcionalidad de base 12 , donde se utilizan las relaciones: de igualdad 1/1, la 1/2 dupla o diapasón, la $2 / 3$ sesquiáltera o diapente y la de $4 / 3$ sesquitércia o diatesarón.

\section{La construcción de la fábrica gótica}

La catedral de Tortosa (fig. 5), dispone de un ábside con doble deambulatorio ejecutado entre 1347 y 1435 . La primera fase fue la construcción del cinturón de las capillas radiales, construidas de forma correlativa entre (1383-1424) y con las claves rematadas a 45 palmos y de proporción $9 / 5$, en términos de despliegue de la sección gótica. En la segunda se construye el deambulatorio, cubierto a 72 palmos, donde las bóvedas fueron construidas simétricamente (1424-1435), desde la nave hacia el interior del presbiterio. Finalmente se cubre el presbiterio (1435-1441), con una clave de 10 palmos de diámetro, situada a 100 palmos del suelo (fig. 6). Tanto el deambulatorio como el presbiterio tienen un proporción de 9/6.

En el inicio de la obra el maestro impone la unidad de medida, una cana de ferre per pendre mesures de l'obra (Ll.o.2 1345-1347, f.36 v) ${ }^{36}$. El patrón básico de las medidas de los Llibres d'Obra (ACTo), es la cana de Tortosa definida en el Libro IX, Rubrica 15.5 de las Consuetudines Dertosae (1272) (AHCTE cod.53, fol.256r) ${ }^{37}$, tiene $185,8 \mathrm{~cm}$ y el palmo de $23,23 \mathrm{~cm}$. Así el conjunto del ábside de la catedral tiene unas proporciones metrológicas: 150 palmos de ancho y 100 de fondo y altura. Las capillas radiales, de planta cuadrada, tienen interiormente $21 \times 21$ palmos. Los puntos de los pilares del ábside, donde se replanteo la obra, equidistan 24 palmos, 3 canas de Tortosa, replanteados a 54 palmos del centro del presbiterio. Existe así, una relación entre el radio de la circunferencia (54 palmos) 18 módulos, y el lado del polígono de catorce lados (24 palmos) 8 módulos, estableciendo una proporción de 9/8.

La traza de Guarc (c.1345-1380) y el replanteo general de fábrica (1383-1424) tienen la misma estructura genética (fig.7). La solución entre el radio, 18 módulos de la circunferencia del deambulatorio, y 8 módulos de la capilla radial, y que es además el lado del doble heptágono, establece una solución geométrica y a la vez aritméticamente metrológica. En la fábrica, para construir una capilla de 3 canas ( 24 palmos), se necesita un radio para el deambulatorio de 6 canas y 6 palmos (54 palmos).

Dada la estructura modular del pergamino de Guarc, se permite especular con una relación aritmética que sea capaz de trazar el cimborrio. De esta manera se puede hacer conmensurable la medida del octógono, de la misma manera que lo hizo la Metrica (LI.XVIII) de Herón Alexandria (c.20-62) ${ }^{38}$, utilizando la relación de 12/10 entre el radio y el lado del octógono. Esta teoría ha sido aplicada al trazado de templos y ábsides de planta octogonal ${ }^{39}$. En el caso de Guarc, se podría replantear el cimborrio sobre el cuadrado $18 \times 18$, donde el lado del octógono fuera igual al fondo de la capilla radial 7,5 módulos, y que tiene 8 módulos de ancho, cumpliéndose así la condición heroniana (fig.8).

\footnotetext{
36 Almuni, 1991: 214.

37 Felipe II (1527-1598), Cortes De Monzón (1585). Capitulo 89. AHCTE, 387 (Comú II-63).

38 Schoene, 1903: 57-59. Bruins; 1964:3).

39 CANTor, 1907: 377-379. ÖZdural, 2002: 217-242. Svenshon,2008:103-112. Høyrup, 2009: 367-377.
} 


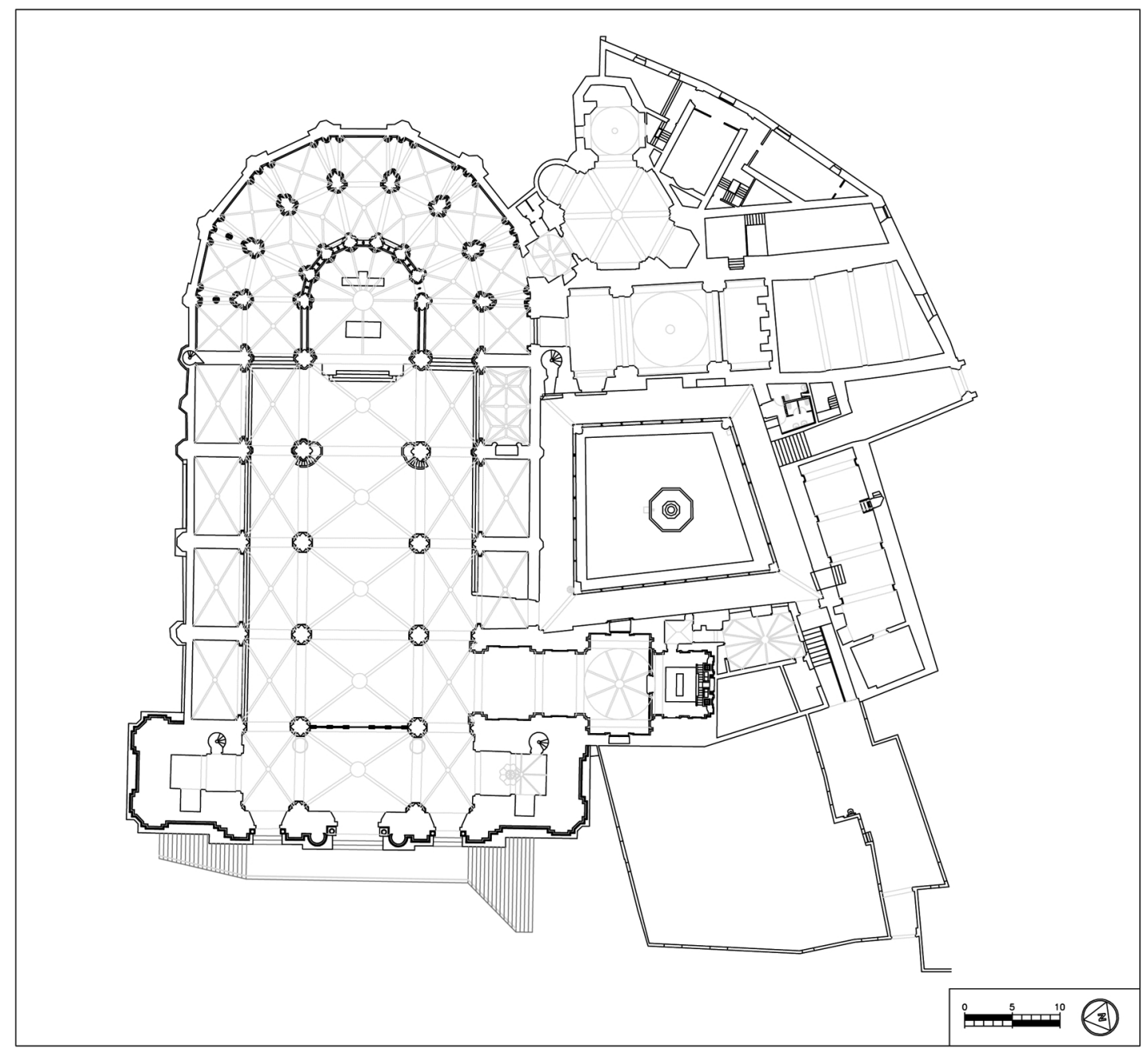

Fig. 5. Planta catedral Santa María Tortosa.
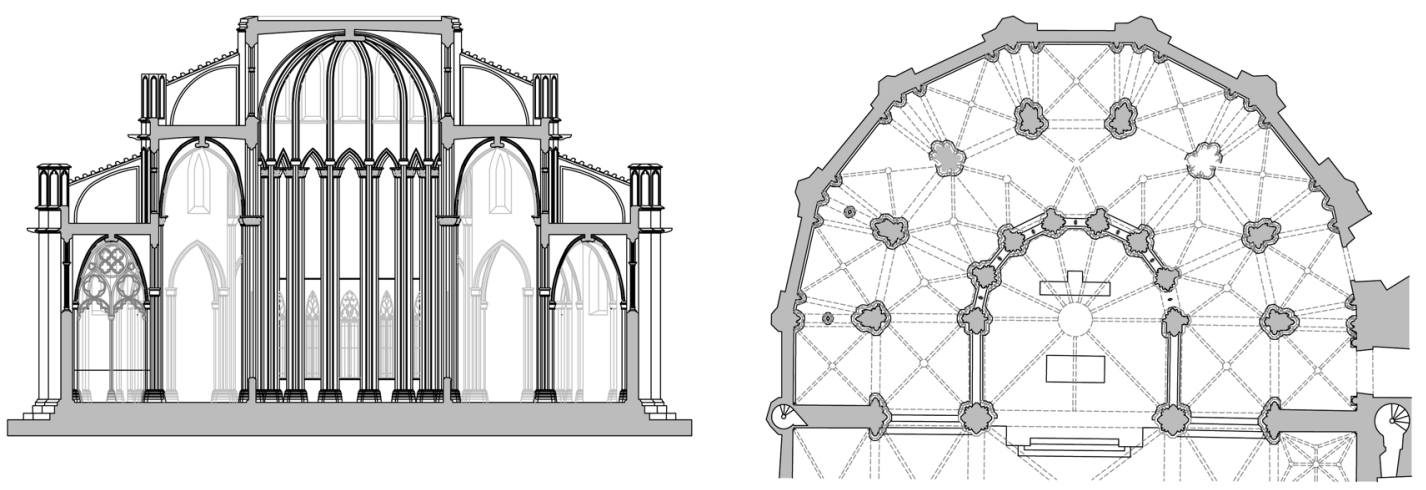

Fig. 6. Planta y sección ábside catedral Tortosa.

Arch. esp. arte, LXXXVII, 348, OCTUBRE-DICIEMBRE 2014, 321-334 ISSN: 0004-0428, eISSN: 1988-8511, doi: 10.3989/aearte.2014.20 

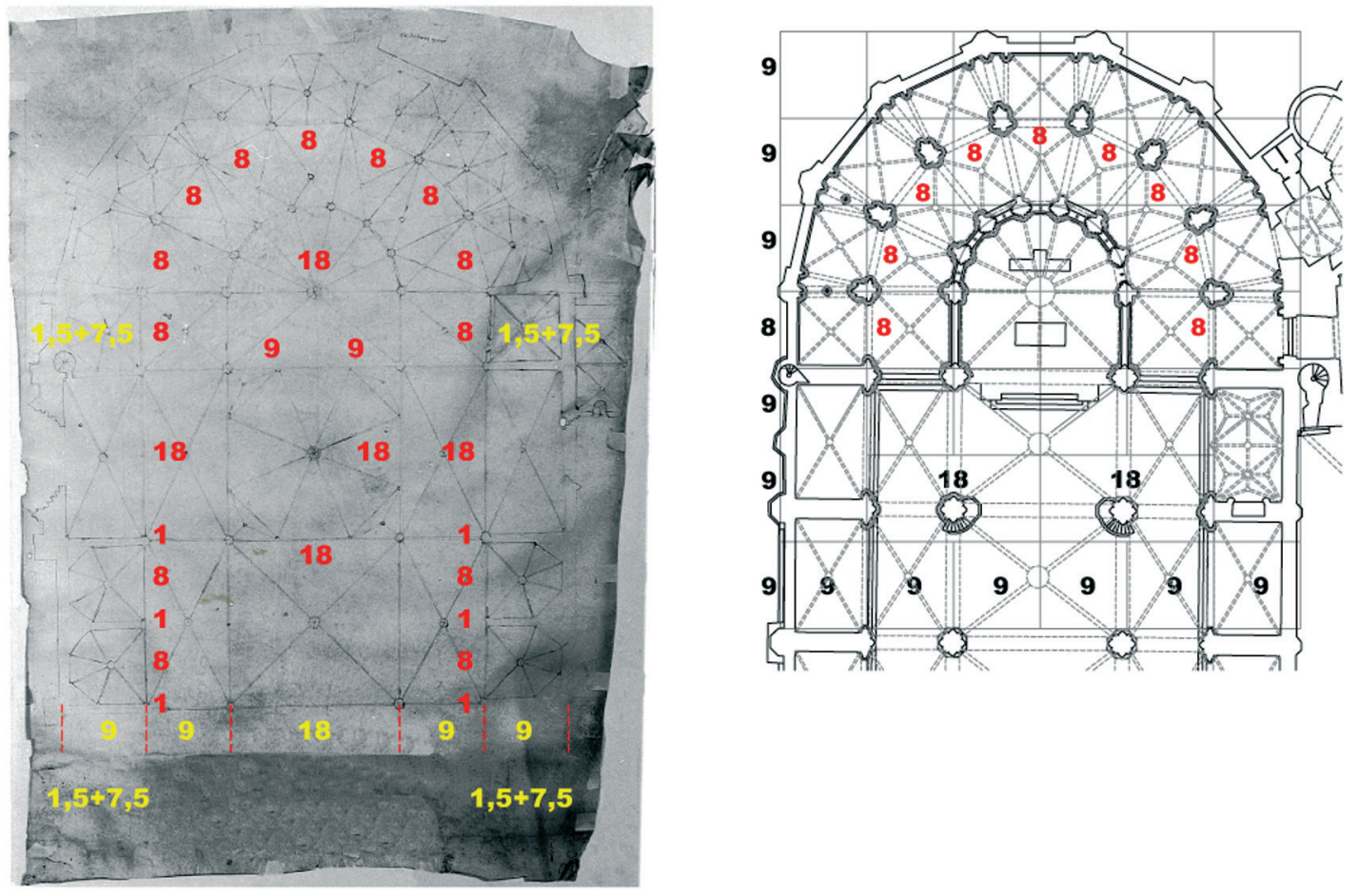

Fig. 7. Construcción proporcional de la Traza Guarc (c.1345-1380) y del ábside catedral de Tortosa (1383-1441).

\section{Traza y proporcionalidad en la catedral de Tortosa}

Marciano Capella (ACTo 80), plantea dos tipos de líneas rhētós, y álogos. Tanto en el trazado del heptágono empleado por Guarc, como en el trazado del ábside, se utiliza la relación entre el ancho de la nave 9/8 con la capilla lateral. Con ello se hace conmensurable, e iguales, las capillas del tramo recto del ábside y las situadas en la cuerda de ábside. Esta mediada en términos de Capella es de una línea rhētós. Por otra parte la relación de Guarc, 9/8, se interpreta en Calcidio ACTo 80 y Marcobio ACTo 236, como la parte y su octava $(1+1 / 8)$, a la que definen como epogdo. El trazado geométrico del octógono de Guarc (c.1345-1380) es similar al que se publicará en la Geometrie Deutsch (1486). Su trazado es álogos, inconmensurable, puesto que abate la diagonal del cimborrio. La transposición aritmética del octógono puede tener la base de la Metrica de Herón de Alejandría. Ésta permite trazar, sobre un cuadrado de base 18 módulos de cimborrio, un lado de medida 7,5 módulos, igual que el fondo de capilla lateral, convirtiéndose así en una media de línea rhêtós.

La relación de Guarc 9/8 no aparece en los tratados cultos. Puede ser así un instrumento de la geometria fabrorum, que permite a la vez una solución geométrica y aritmética. En la catedral de Tortosa, se construye a partir de la medida de la capilla, 3 canas (24 palmos). La medida se despliega a manera de algoritmo, mediante números enteros, determinando así todas las medidas del ábside. Las grandes medidas de la catedral de Tortosa disponen una proporcionalidad basada en modulaciones numéricas, diatesarón y diapente, que bien conocían los canónigos a través de los códices ATCo 80 y ACTo 236. 
(1)

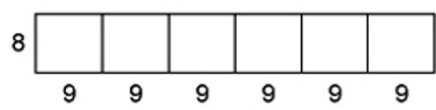

(2)

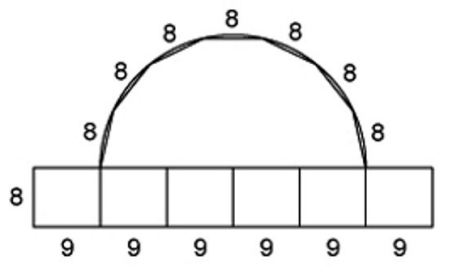

(3)

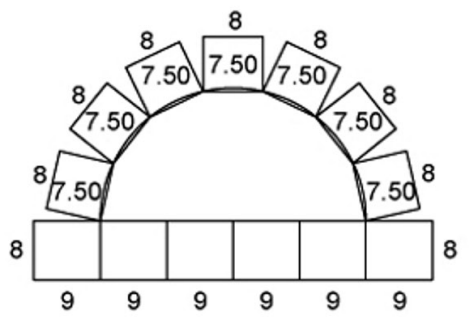

(4)

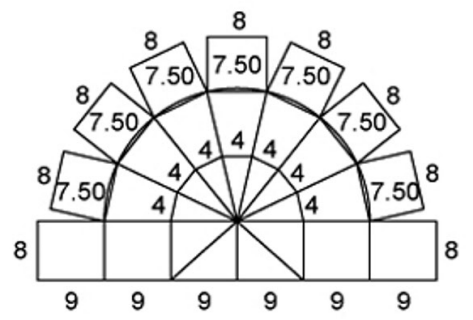

(5)

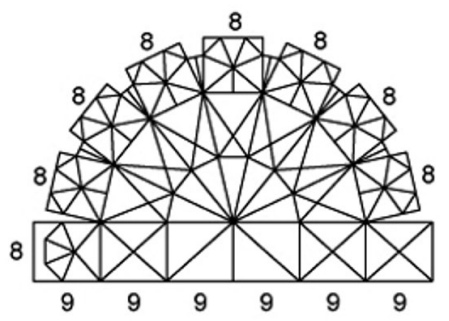

(6)

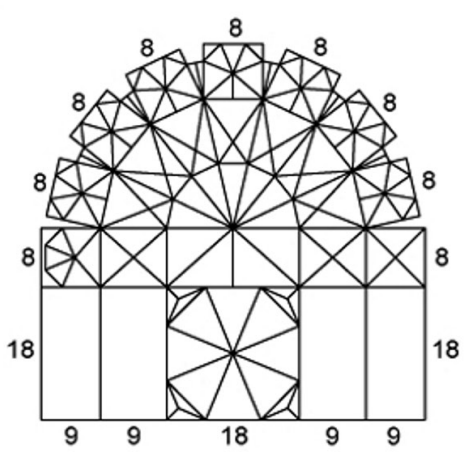

(7)

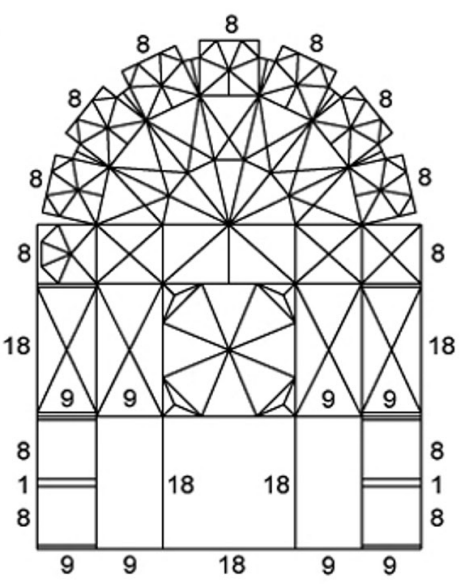

(8)

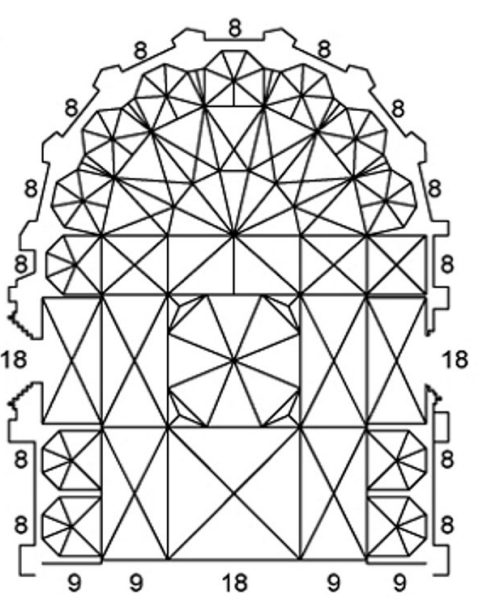

Fig. 8. Estructura proporcional en la traza de Guarc.

Arch. esp. arte, LXXXVII, 348, OCTUBRE-DICIEMBRE 2014, 321-334

ISSN: 0004-0428, eISSN: 1988-8511, doi: 10.3989/aearte.2014.20 
El concepto de proporcionalidad como ciencia se difundió en la Europa gótica, por el De Scientiis de Domingo de Gundisalvo (fl. 1150). Su precedente es el Catálogo de las ciencias de al-Fārābī (c.870-950). La matemática, sciencia doctrinali, dice al-Fārābī, es una de las cinco ciencias que se conocen. Comprende la aritmética, geometría, la óptica, la astronomía, la matemática, la música, la ciencia de los pesos y la ingeniería. En la Aritmética y la Geometría tienen las bases y principios todas las demás ciencias matemáticas, diferenciando en estas disciplinas entre theorica y practica ${ }^{40}$. Gundisalvo utilizará estos mismos términos en el De Scientiiis ${ }^{41}$, y divulgados en Speculum Doctrinale al mundo de las catedrales góticas por Vicente de Beauvais $(\text { c.1194-1264 })^{42}$. La dualidad theorica y la practica impregnará la definición de las ars medievales. El concepto está también latente en la liturgia del Prochiron, vulgo rationale divinorum officiorum (1291), de Guillermo de Durando (1230-1296) ${ }^{43}$. En ella se define la correspondencia entre la ecclesia materialis y la ecclesia spiritualis. ${ }^{44}$. La catedral medieval ecclesia materialis visualiza el hecho constructivo de la fábrica, acogiendo la ecclesia spiritualis. La belleza de la construcción dependía de la habilidad de los maestros medievales, con su practica que es activa. Frente a ella, el conocimiento de los promotores de la catedral, teorica que es speculativa.

\section{BIBLIOGRAFÍA}

Agustín, S. Obras Completas de San Agustín XVI. La Ciudad de Dios (1. ${ }^{\circ}$. (Madrid: Biblioteca Autores Cristianos, 2000).

Agustín, S. Obras Completas de San Agustín XVI. La Ciudad de Dios (2. ${ }^{\circ}$. (Madrid: Biblioteca Autores Cristianos, 2001).

Almuni, V. L'Obra de la Seu de Tortosa (1345-1441). (Tortosa: Cooperativa Gràtica Dertosense 1991).

Almuni, V. La catedral de Tortosa als segles del gotic. 2 Vols. (Barcelona: Fundació Noguera 2007).

Almuni V.; Lluis J. La traça de la catedral de Tortosa. Els models d'Antoni Guarc i Bernat Dalguaire. Lambard. Estudis art medieval, (IX) (1997), pp. 312-313.

Almuni V; Lluis J. Sancta Maria Dertosae. Catedral de Tortosa. Guia Històrica i Descriptiva. Tortosa. (Capítol Catedral de Santa Maria 2000).

Alonso B.; Jiménez A. La Traça de la iglesia de Sevilla. (Sevilla: Cabildo Metropolitano, 2009).

Alonso B.; Jiménez. A. La traza guipuzcoana de la catedral de Sevilla. Actas del Sexto Congreso Nacional de Historia de la Construcción (2009b). Valencia, 21-24 octubre 2009, pp 63-74.

Alonso, M. Domingo Gundisalvo. De Scientiis. Compilación a base principalmente de la de Al-Farabi. (Madrid: Consejo Superior de Investigaciones Científicas, 1955).

Arroyas M., Zaragozà, A. Una arquitectura gòtica mediterrània. (Valencia: Generalitat Valenciana 2003).

Ascani, V. Le dessin d'architecture médiéval en Italie. En: Recht (ed Les bâtisseurs du Moyen-Âge. (Strasbourg: Editions les Musses de la Ville de Strasbourg 1989), pp.255-277.

Ascani, V. Il Trecento disegnato. Le basi progettuali dell'architettura gotica in Italia. (Roma: Viella, 1997).

Bayerri E. Los Códices Medievales de la Catedral de Tortosa. Novísimo inventario descriptivo. (Tortosa: Talleres Gráficos Algueró y Baiges, 1962).

Beaujouan, G. Réflexions sur les rapports entre théorie et practique au Moyen Age. En J. E. Murdoch and E.D.Sylla (ed.). The Cultural Context of Medieval Learning. P. 437-484. (Dordrech: Reidel Publishing Company, 1975).

\footnotetext{
40 GoNZÁLEZ, 1932: 97-105.

41 Alonso, 1955: 85-112.

42 Alonso, 1955: 143-167. Traducción edición (1591).

${ }^{43}$ Códice ACTo 58, Incunables ACTo 258, Roma (1477) y ACTo 290, Venecia (1482).

44 SEBAStiÁn, 1994: 352-355.
} 
Bechmann, R. Villard de Honnecourt. La pensée tecnique au XIIIe siècle et sa communication. (Paris: Picard Éditeur, 1993).

Bork, R. The Geometry of Creation: Architectural Drawing and the Dynamics of othic Design. (Farnham: Ashgate Press, 2011).

Bouvelles, Ch. Liber de intellectu, Liber de sensibus, Libellus de Nihilo, Ars oppositorum, Liber de generatione,... Libellus de Mathematicis rosis, Liber de mathematicis corporibus, Libellus de mathematicis supplementis. (Paris: Henri Estienne, 1510).

Bruins, E.M. Codex Constantinopolitanus Palatii Veteris No. I, Janus Suppl. 2, 3 vols. (Leiden: Brill. 1964)

Bubnov. N.M. Gerberti postea Silvestri II papae opera mathematica (972-1003). (Berlin: Friedländer, 1899).

Bucher, F. Design in Gothic Architecture. A Preliminary Assessment. Journal of the Society of Architectural Historians, vol XXVII (n. ${ }^{\circ}$ ), March 1968, pp.49-71.

Bucher, F. Medieval Architectural Design Methods, 800-1560. Gesta, 1972, Vol.11, No.2. P.37-51.

Bucher, F. Architector.The Lodge books and sketchbooks of medieval architects. Vol. I. (New York: Abaris Book 1979).

Cantor, M. Vorlesungen Uber Geschichte Der Mathematik V1, Part 2. (Leipzig: B.G. Teubner,1907).

Casaseca, A. Trazas para la catedral de Segovia. Archivo Español de Arte, LI, 1978, pp. $29-51$.

Curtze, M. Jordani Nemorarii Geometria vel de triangulis libri IV. Mitteilungen des Coppernicus-Vereins zu Thorn 6/1887.

Durero, A. Underweysung der Messung, mit dem Zirckel und Richtscheyt: in Linien Ebnen vo gantzen Corporen (Nürenberg: Hieronymum Formschneyder, 1525).

González, Á. Alfarabi 870-950. Catálogo de las ciencias. (Madrid: Instituto Miguel Asin. CSIC, 1954).

Hiscock, N. The Wise Master Builder. Platonic Geoemtry in Plans of Medieval Abbeys and Cathedrals. (Aldershot y Brookfield, Vermont: Ashgate, 2000).

Horn, W; Born, E. The "Dimensional Inconsistencies" of the Plan of Saint Gall and the problem of the Scale oft he Plan. The Art Bulletin (1966). Vol. 48, N.3/4, pp.285-308.

Horn, W; Born, E. The Plan of St. Gall. A study of the architecture \& economy of, \& life in a paradigmatic carolingian monastery. 3 Vol. (Berkeley: University of California 1979).

Hoffstadt, F. Principes du Style Gothique. Exposés d'après des documents authentiques du moyen Âge avec 40 planches in-folio. A l'usage des artistes et des ouvriers. (Paris: A. Franck et Victor Didron 1847).

Høyrup, J. The Rare Traces of Constructional Procedures in 'Practical Geometries. En Horst Nowacki \& Wolfgang Lefèvre (eds), Creating Shapes in Civil and Naval Architecture. (Leiden \& Boston: Brill, 2009), pp.367-377.

Lassus, J.B. Album de Villard de Honnecourt. Architecte du XIIIe siècle. (Paris: Imprimerie impériale, 1858).

Lavedan, P. L'architecture Gothique Religieuse, en Catalogne, Valence et Baléares (Paris: Henri Laureen. 1935).

Lluis i Ginovart, J. "Una breu reflexió de l'Àbsis de la Catedral de Tortosa". La Veu del Baix Ebre n. ${ }^{\circ} 1.405$, 1984-31-8: 25-26.

Lluis i Ginovart, J. Traça de la catedral de Tortosa. En Fidei Speculum (ed.), Art litúrgic Diócesi de Tortosa. (Barcelona: Fundació "la Caixa" 2000).

Lluis i Ginovart, J. "Traza del ábside de la catedral de Tortosa”. La Llum de les Imatges. Sant Mateu 2005. (Valencia: Generalitat Valenciana. 2005), pp 240-.

Matamoros, J. La catedral de Tortosa. Trabajos monográficos acerca de su construcción y de su contenido artístico y religioso (Tortosa: Editorial Católica 1932).

Muñoz, I. Manifiesto geométrico, plus ultra de la Geometría práctica, adicional al IV libro de Euclides, construcción y demostración geométrica del Triangulo Isósceles, propio del heptágono regular, y descripción de la misma figura. (Bruselas: Francisco Foppens, 1684).

Nilsen, D. The Squaring of the Circle in two Early Norwegian Cathedrals? Nexus Network Journal. April 2001, Vol. 3, I. 1, pp 27-42.

Özdural, A. The Church of St. George of the Latins in famagusta: A Case Study on Medieval Metrology and Design Techniques. En: WU, N. Ad Quadratum. The Practical Application of Geometry in Medieval Architecture. (Burlington: Ashgate (2002). P. 217-242).

Raynaud, D. Abū al-Wafă' Latinus.? A study of method. Historia Mathematica 39, 2012, pp.34-83.

Arch. esp. arte, LXXXVII, 348, OCTUBRE-DICIEMBRE 2014, 321-334

ISSN: 0004-0428, eISSN: 1988-8511, doi: 10.3989/aearte.2014.20 
Recht, R. Le traité de Géométrie de Mathieu Roriczer. En: Les bâtisseurs du Moyen-Âge. Histoire et Archéologie. Dossiers (1980). Noviembre, pp. 24-25.

Recht, R. (Ed.). Les batîsseurs de cathédrales gothiques. (Strasbourg: Musées de la ville de Strasbourg, 1989).

Recht, R. Le Dessin d'architecture. Origine et fonction. (París, Hazan, 1995).

Roriczer, M. Das Büchlein von der Fialen Gerechtigheit (fak. Regensburg 1486) und Die Geometria Deutsch (fak. Regensburg un 1487/88). (Regensburg: Hürtgenwald Guido Pressler, 1999).

Ruiz de la Rosa, J. A. (1987a). Geometría fabrorum o la antitesis de las teorías sofisticadas. Boletín Académico de la Escola Técnica Superior de Arquitectura da Coruña, 7 (Octubre): 52-59.

Ruiz de la Rosa, J. A. (1987b). Traza y simetría de la arquitectura en la antigüedad y medievo. (Sevilla, Universidad de Sevilla).

Sánchez, F. M. Martín de Solórzano: la influencia de Santo Tomás de Ávila en los proyectos constructivos de la Catedral de Coria. Norba-Arte (1982) n. ${ }^{\circ} 3$, pp. 63-76.

Schoene, H.Heronis Alexandrini opera quae supersunt omnia. Vol III: Rationes dimetiendi et commentatio dioptrica. (Leipzig: Teubner. 1903).

Sebastián S. Mensaje Simbólico del Arte Medieval. Arquitectura, Liturgia e Iconografía. (Madrid: Ediciones Encuentro, 1994).

Svenshon, H. Schlag' nach bei Hheron. Der Turm der Winde im Sspiegel antike Vvermessungslehre. Koldewey-Gesellschaft Vereinigung für Baugeschichtliche Fforschung E.V. Bericht über die 45. Tagung für Ausgrabungswissenschaft und Bauforschung. Von 30 april bis 4 maig 2008 in Regensburg, pp103-112.

Vandevyvere, H. (2001). Gothic Town Halls in and around Flanders, 1350-1550: a Geometrical Analysis. Nexus Network Journal. September 2001, Vol. 3, I. 2, pp 59-84.

Vrijs, A. Catalogue. En: Recht (ed). Les bâtisseurs des Cathédrales Gothiques. (Strasbourg: Editions les Musées de la Ville. 1989): 330-483.

Waszink, J.H. Plato Latinus. IV. Timaeus, a Calcidio translatus commentarioque instructus, edit J.H. Waszink, editio altera. (Londinnii, Leidae: En aedibus Instituti Warburgiani, 1975).

Willis, J. Macrobius. Comentarii In Somnium Scipionis. (Stugar-Leizpzig: B.G. Teubner,1970).

Willis, J. Martianus Capella, edidit J. Willis. (Leipzig: Teubner, 1983).

Woepcke, M. F. "Recherches sur l'historie des Sciences mathématiques chez les orientaux, dápres des traités inedits Arabes et Persans. Deuxième siecle. Analyse et extrait d'un recueil de constructions géométriques par Abûl Wafa". Journal asiatique. (1855). Fevier-Avril. Vol.V, pp. 218-256:309-359.

Fecha de recepción: 17-V-2013

Fecha de aceptación: 03-III-2014 